\section{A Ierture}

$$
\text { ox }
$$

\section{PARTIAL PYLORIC OBSTRUCTION.}

Being an ABstract of the Third of the James Watson Lectures on "SOMe Chronic Gastric Disorders." * BY

\section{A. ERNES'T MAYLARD, B.S.LoND., F.R.F.P.S.GLASG.,} F.R.S.EDIN.

CONSULTING SURGEON TO THE VICTORIA INFIRMARY, GLASGOW.

THE causes of pyloric obstruction may be primarily classified into anatomical and pathological; and the latter may be subclassified into (1) those dependent on inflammation and (2) those the result of new growths.

\section{Anatomical Obstruction.}

\section{Congenital Narrowness of the Pyloric Orifice.}

This class has receired but comparatively slight recognition, but I trust I may be able to show that it plays no inconsiderable part in the group of conditions that obstruct the gastric outlet.

At an early period of my work, when operating for obscure and intractable symptoms indicative of gastric derangements, I now and again encountered a condition of the pyloric orifice which I was at a loss to explain on any other ground than that the opening was too narrow to allow of a free and easy exit of the gastric contents; that this was the source of the chronic gastric disorder received confirmation from the fact that relief and cure followed on the operation of gastro-jejunostomy. After an experience of 7. cases I read a paper before the Clinical Society of London ${ }^{1}$ entitled "Congenital narrowness of tine pyloric orifice." Later, in another paper published in the British Medical Journal, ${ }^{2} 12$ other illustrative cases were given. Dr. William Russell ${ }^{8}$ of Edinburgh also contributed an exhaustive article on the subject, published in the same number of the JouRNax, strongly supporting the view that I had promulgated, that such an anatomical condition existed, and accounted for the symptoms met with in certain classes of cases.

Before describing the particular features peculiar to this form of obstruction, I ought perhaps to state what may be considered the normal condition of the orifice as met with by the surgeon on the operating table. Under com. plete anaesthesia the gastric parietes are more or less flaccid. Externally the pylorus can usually be recognized by the somewhat deeper colour it presents in contrast to the neighbouring parts, and by the convergence of vessels, mostly veins, at its lower margin. Only the faintest constriction is, as a rule, visible, the duodenum often passing so imperceptibly into the stomach that it is only by the tactile sensation afforded by the sphincter and the dis. tribution of the vessels that the exact seat of the pylorus is determined. 'There is no constancy in the amount of induration (if I may so express it) which the pylorus presents, a fact which seems to suggest that although the patency of tile canal may be quite normal, there may be considerable differences in the developmental condition of the sphincter-differences possibly dependent on the same physical causes which may naturally affect other parts of the body. I have little doubt that this variation, when not taken into due account, has led to the difficulty experienced by some in determining whether the greater thickness met with in one case as compared with another is or is not due to some excessive liypertrophic or inflam. matory cause. I am, however, convinced that the variation is quite normal, and may exist within considerable linits. Regarding the calibre of the pyloric orifice, I have always found that while the index finger passes easily, it has conveyed to it the sense of a uniformly narrow, elastie ring, which feebly embraces and rolls ofez the finger as it is gently pushed on into the duadeisum. I should consider 12 to $15 \mathrm{~mm}$, the normal calibre duríng life and under relaxation. If a section of the lorus is looked at-such, for instance, as is given in Quain's Anatomy-a sensation such as that just described is what might be reasonably expected. A uniform ring-like projection of

* Delivered before the Royal Faculty of Physicians and Surgeons of Glasgow, Narch, 1920. mucous membrane is seen containing within its reduplicated folds bands of muscle fibre.

When, in my earlier cases, I first met with this ab. normally narrowed condition of the canal, I was inclined to believe that, if not due to chronic spasm, it was the direct result of the stimulating and exciting influence evoked by the introduction of the finger into the pyloric orifice; that such an indigestible morsel as the index finger, so large, solid, and unfit to pass out of the stomach, was certainly likely to cause contraction of the pyloric sphincter whose chief function is to prevent all but the most efficiently peptonized food passing out of the stomach. However, I had on one occasion a very striking and informing illustration of what the parietes of the stomach and the pylorus are like under contraction. The stomach had been opened for inspection and partially withdrawn from the abdomen with the pylorus in view. The patient seemed somewhat suddenly to come out of the anaesthetic, when a violent contraction of the gastric parietes took place, causing a little of the clear fluid in the stomach to be forcibly spurted out. Immediately there followed contraction of the pylorus, which assumed the appearance of a rigid structure about 2 in. in length, and about the circumference of the ring finger. As the patient again came under the influcnce of the anaes. thetic the parts relaxed; and it was under this relaxed state that I felt a narrow, uniform, ring-like constriction of the pyloric aperture incapable of admitting the apex of the index finger. It was the information gained in this particular case that led me first to doubt whether these narrowed orifices could be the result of a chronic spas. modic condition, and to suspect that they owed their existence to some abnormality in development rather than to any acquired cause.

As to the nature of this abnormality, I am inclined to regard it as due to an excess of reduplication of the pyloric fold, whereby the width of the valve is increased, but the calibre of the canal proportionately diminished; that it is, therefore, purely anatomical and congenital in origin. Such a definition entirely fits the facts revealed in the cases met with, for the obstruction was al ways perfectly uniform, and simply gave the impression that the orifice was abnormally small; the smaller it was the greater the resistance it offered to the introduction and passage of the finger. Further, it shonld be emphasized that the resistance was in no sense like that encountered as the result of cicatrization. That, as is well known, rarely gives way, except as the result of a certain amount of laceration of newly formed tissue; while, in the majority of these. cases-the exception being those of a very high degree of narrowing-the ring gradually dilates, and uniformly stretches over the advancing finger.

If I lave been right in the construction I have put forward regarding the nature and origin of this narrowing of the aperture, it is possible to conceive how we may have, solely as the result of development, any calibre of the pyloric orifice between that which we regard as normal and that which will admit only a small sized-say No. 6 -ordinary urethral catheter.

I ought to say, befcre proceeding further, that, although ignorant of the fact when I first met with the condition just described, subsequent investigation has brought to my notice the description of this same appearance of the part by others. Landerer (quoted by Maier ${ }^{4}$ ) some years ago seems to have been the first to draw attention to this simple narrowing of the pylorus unaccompanied by any marked thickening or other changes connected with the part. Maier discusses this congenital abnormality; his cases. numbering twenty-one, were necropsies on patients who had died mostly from other causes than those in any way associated with the gastric condition. For some ycars, therefore, the possibility of chronic gastric disorders in the adult dependent on this particular lind of narrowing of the pyloric aperture has been recognized; but it is only comparatively recently, since the stomach has been explored by the surgeon, that the cause and its effects have come to be more exactly noted, and still further treated. The subject is briefly referred to by Mayo-Robson and Moynihan in their book, ${ }^{5}$ where a very typical case illustrative of the condition is recorded. as

If further support of the opinions expressed were neased I think it is to be found in the unmistakable evidence generally acknowledged to exist in some other parts of the 
body possessing somewhat similar functions. Thus, there are distinct variations in the calibre of the ileo-caecal orifice; and William Mayo ${ }^{6}$ has reported cases be has operated upon in which an abnormal narrowing of the passage between the ileum and the caecum has been the cause of constipation. No evidences of disease of any kind were discoverable to account for the smallness of the valvular aperture; presumably, therefore, the condition had to be regarded as one due to defective development of the ileo-caecal valve. The uterine cervical canal affords yet another analogy, for the passage may be congenitally narrow and prove obstructive to the easy and unimpeded flow of the menses. It is not, perhaps, overstretching the comparison to indicate the great differences that exist in the size of the oral aperture, and in the width of the palpebral fissure, not that any undue smallness of either of these apertures is any impediment to the particular part each has to play in the human economy, only they serve to show, from the obvious natural existence of developmental variations, that any such defect at the pyloric aperture is a perfectly reasonable assumption.

Hypertrophic Stenosis of the Pyloric Orifice.

Oen of its cause or origin is hypertrophic stenosis of the pyloric aperture. Although a condition most frequently met with in early life, it is not entirely confined to this period, as cases are occasionally encountered in the adult; and I have myself met with two unmistakable illustra tions. Maier ${ }^{4}$ fully established its existence in later life in some of the cases he describes. Others, too, in this country, among whom may be specially mentioned Clinton Dent and E. Cautley, ${ }^{7}$ have likewise confirmed the existence of this particular form of obstruction in the adult.

\section{Pathological Obstruction}

1. Chronic Ulceration.

Of all the causes that give rise to obstruction none are so frequent as those which arise from chronic ulceration of the non-malignant type. The obstruction is partly due to cicatricial contraction, but more often it is the result of the inflammatory thickening associated with the active spread of the ulcer. So great sometimes is this hard, more or less oedematous mass, that it is mistaken for a malignant growth.

The variable phases of the pathological aspect of the subject all have their bearing on the multiple manifesta. tions which the lesion may present. For, as will be pointed out, it is the capacity or incapacity of the stomach to overcome the obstruction with ease or with difficulty that leads to the many complexities in symptomatic mani festations which so frequently complicate the diagnosis of this particular class of cases.

\section{Newv Growths.}

The new growths which may cause obstruction are both malignant and innocent. The latter may be bricfly dismissed with a few words. Occurring on the inner surface of the stomach close to the pylorus, they may block the aperture like a ball-valve. External pressure is another rare cause of obstruction, and may be due to a distended gall bladder or a mass of enlarged glands over the head of the pancreas. By far the commonest form of new growth is carcinoma. This may commence in the walls of the canal, or be the result of invasion from without.

\section{SYMPTOMS.}

As regards the symptoms of pyloric obstruction, it will simplify matters very materially if we first consider what ire likely to be the natural physiological and pathological sequiences of any hindrance to the escape of the contents of the stomach into the duodenum. I do not intend to continue the discussion into a consideration of those adranced cases in which there is no difficulty in diagnosis -where, for instance, we have all the signs of a stomach that occupies a considerable portion of the abdomen, holds several pints of fluid, and retains so much of the food taken that the patient occasionally vomits a "basinful" or "chamberpotful" of sour, incompletely digested, porridge-like material containing fragments of food taken several hours or days before. Fortunately we rarely see these. advanced cases now. It is not, then, with the symptoms of this type of case that I propose to deal, but with those in which the stomach is still capable of overcoming the obstruction, though with variable degrees of success. For want of a better and more accurate term to apply to these cases they may be designated cases of partial pyloric obstruction. It is the variableness of the amount of obstruction that constitutes the difficulty so frequently encountered in diagnosis.

If we consider the possible physiological effects of ob. struction at the gastric outlet-and by this it must be understood that not only the pylorus itself, but the parts of the canal both in front of and distal to it are includedit will be clear that the organ first to be affected will be the stomach itself. Whatever be the relative importance of the different functions of the stomach, it is certain that nothing can talse the place of the motor function; and the contents of the stomach-however much they may be acted upon, whether sufficiently or not, by the gastric juice-must be propelled out of the viscus by the contraction of the muscle tunics. Thus, then, it may be reasonably assumed that the primary effect of obstruction will be to place increased work upon the muscle layers; and the amount of work which they are called upon to perform will be in direct proportion to the amount of obstruction that they have to overcome. It is more than likely that the increased pressure to which the contents of the stomach are subjected, in the greater effort of the organ to rid itself of its digested ingesta, may deleteriously affect the normal secretion of the gastric juice both quantitatively and qualitatively. But, however this may be (and possibly it is only a matter of moment in cases of considerable and prolonged obstruction), it is the effect upon the muscle tunics that determines the interesting symptomatic vagaries of the condition-the factor deserving of most attention.

Consider, for a moment, the parallel illustration of any voluntary muscle of the body which is called upon to undergo prolonged exertion. If the work to be done be of an intermittent type, the periods of rest and increased requirements lead to a compensatory development, which, up to a certain pitch, succeeds in accomplishing the ends required. If, however, there be no periods of rest interposed, weariness intervenes, and an entire failure of accomplishment ensues. Introduce another factor into the scheme-failure to supply the body with proper and sufficient nourishment-and muscle inertia will be rapidly experienced.

'The stomach does for a time, and in direct proportion to the amount of obstruction it has to overcome, succeed iil carrying out its normal functions. Its muscle tunics hypertrophy, and, in consequence, it is enabled to drive out its contents successfully; but, as time goes on, it becomes more and more susceptible to influences which may reach it either by way of the nervous system or the blood supply. Thus, causes of various kinds, which arise from nerve exhaustion or imperfect metabolism affecting the constitution as a whole, influence the struggling stomach in particular; muscle inertia then ensues, and instead of the viscus propelling its contents through the obstructed oritice, they remain, only in part it may be, pent up in the gastric cavity, awaiting the time when the muscle parietes shall have regained sufficient energy to effect total expulsion. The sequence of events, however, may not prove so simple as thus far they may appear. For when, for the reasons given, the stomach is unable to expel its contents, it may, though sheer weariness of effort, relax its endeavour and give way to passive dilatation. To what extent this may go, and how long it may last, will again depend on the nature and degree of obstruction, and the general physical state of the patient. Herein will be seen to enter the symptomatic vagaries of the disease; for there is no telling when the symitoms will subside, exactly why they should appear, and why they are of the nature manifested. And yet, talking these considerations collectively, they furnish the best clues to the nature of the complaint.

Let me now ask for a closer investigation into some of the symptoms which the above considerations naturally lead one to expect will at one time or another manifest themselves.

1. In the first place, there is usually nothing about the general appearance of the patients to suggest constitutional disease; in other words, the gastric symptoms appear to be solely dependent on the disturbances affecting the viscus itself : indeed, there is often an appearance 
of health and robustness about them, and between their "attacks" they are quite well. In those cases where the patients are thin, it is not the thinness of constitutional disease, but simply an emaciation due to lack of sufficient nourishment.

2. The earlier history of the case will sometimes indicate that the initial symptoms suggested an active ulcerative attack; but often any such sudden onset is absent, and the symptoms begin and creep on quite jnsidiously. This is usually the course taken by that type of obstruction dependent on congenital narrowness of the pyloric orifice.

3. Exacerbation of the symptoms is engendered by anything that has an enfeebling effect upon the constitution. If from undue exertion, mentally or bodily, from worry or anxiety, the patient is reduced to a state below par, the same diminished sense of physical and physiological fitness is experienced by the stomach and renders it less capable of overcoming the obstruction.

4. It will nearly always be noted that while, at the outset, the attacks of dyspepsia are fow and far between, they gradually increase in frequency; and as they become more frequent so they become more severe. This sequence of events is most suggestive, because it indicates two probabilities-one, an increasing narrowness of the pyloric aperture; the other, the increasing failure of the stomach to overcome the obstruction.

5. Physical examination is only of value as regards dilatation. And here it may be remarked that, as a symptom, no more importance should be attached to the absence than to the presence of dilatation; for while at one time the viscus may be able to overcome the obstruction, at another it may fail; but, notwithstanding its absence, the obstruction is there. Rather, therefore, should it be taken that its presence at one time and its absence at another is an indication of partial pyloric obstruction.

As a means to diagnosis the $x$ rays and opaque meal aid us considerably; but, from all that has been said about the occasional power of the stomach to empty itself com. pletely by overcoming the obstruction, it can be well understood how that sometimes an erroneous impression may be conveyed. If, however, the meal takes an unduly long time to pass out of the stomach, and the skiagram shows, in addition, some irregularity in the shape of the parts in the neighbourhood of the obstruction, the picture presented by the $x$ rays will help to confirm the opinion which the symptoms have probably already suggested.

\section{Treatment.}

If surgery had done nothing more in the treatment of chronic gastric disorders than deal with this particular class of cases, it would have earned for itself a reputation beyond the power of words to express. I know of few patients whose existence may be more miserable, and who, when relief is afforded, become more happy.

The condition of partial pyloric obstruction can always be temporarily relieved by rest in its broadest sense and careful attention to diet; but, as the condition is de. pendent essentially on mechanical causes, it is only by mechanical measures that it can adequately be dealt with. With regard to operation, we have the choice of two primary procedures. One is to deal directly with the prutruction; the other, to leave the obstruction untouched and circumvent it by uniting the stomach to the bowel. As regards the first of these two procedures, much must depend upon the nature of the obstruction. If it be due to growth, removal if possible; if it be of an innocent nature and the part can be freely mobilized, gastro. duodenostomy by Finney's method would appear to be better than either the Heineke-Mikulicz operation of pyloroplasty or pylorodiosis.

As regards the second of the two procedures, short circuiting by the "no-loop" operation of gastro-jejunostomy is the sole method employed. Of all the operations for obstruction none have been more frequently executed than this. In my own wards, for instance, the operation has been performed by myself and by my assistant colleagues, Mr. Grant Andrew and Mr. Farquhar Macrae-now surgeons to the institution-428 times up to the end of 1919. Perhaps the best testimony that can be borne to the permanent good effected by. the operation is to be found in the fact that, to my lnowledge, there lives at the present day a female patient, perfectly well and engaged in active work, whom I operated upon seventeen years ago; and another, a male, also quite well and driving a motor car, upon whom I operated nineteen years ago.

1 The Clinical Society' REFERENCEs.

1he Clinical Society's Transactions, vol..xxxvii. 1904, p. 63; and JOTRNAL, vol. ii, 1908 p 71 , 8 ol. i, 1904, p. 417. 2 BRITISH MEDICAT Band cii, p. 413. 5 Mayo-Robson and p. 68 . 1 Virchcw's Archiv, Stomach, p. 42. 6 Annals of Surgery, vol. xxxii. p. 364. 7 Transaction of the Royal Medical and Chirurgical Society, London, vol. 1xxxvi.

\section{d of extur}

ox

\section{HEART AFFECTIONS IN RELATION TO}

\section{THE LABOUR MARKET.}

Delivered during the Post-Graduate Course at the National Hospital for Diseases of the Heart. BY

R. O. MOON, M.D., F.R.C.P. PHYGICIAN TO THE HOSPITAL.

DURING the war, and particularly in connexion with recruiting, doctors were continually having to estimate the all-round efficiency of a man as a fighting unit, quite apart from having to disqualify him for such obvious disabilities as flat-foot, hammer-toe, hernia, etc.

Into such an estimate the strength and general condition of the heart necessarily entered very largely, and indeed became one of the most important and difficult factors to appraise in deciding upon a man's category. Owing to the vast complexity of modern warfare, in which whole nations are, so to speak, in arms, there came to be numerous posts which could be filled by men who were very far from being Al men.

In the fully organized army a constant process of sorting was always going on by which those with impaired efficiency were moved downwards into lower grades, while those whose efficiency had improved were moved upwards.

There is no reason why in civil life a similar effort, doubtless on a somewhat different system, should not be made for estimating the efficiency of a man in the labour market. The problem here is in one sense easier than in the army, in another sense more complex; for clearly a man might be $\mathrm{Al}$ for his particular trade, but not $\mathrm{Al}$ as an infantry soldier, on whom frequent calls for excessive exertion may be made at any hour of the day or night, so that many a man, though he might only be B1 or B2 from the army standpoint, might quite well be $A 1$ in his own particular occupation, which did not call for such sudden and strenuous forms of exertion, though it could not in any sense be classed as "light work." To quote from American experience :

Throughout the war it remained a strange paradox that the "irritable heart of soldiers," which is a functional neurosis where the heart is known to be intrinsically normal, should give rise to such pronounced cardiac symptoms, whereas the cases of valvular heart disease were so completely free from symptoms; in the "soldier's heart" anything approaching a normal heart rate even during rest is very unusual. It was generally found that mild cases of valvular disease in the arm examinations showed a cardiac response well within normal limits and were remarkably free from subjective thits and were remarkably free from subjective sensations, which such symptoms are usually pronounced.1

We want to consider this afternoon how far cardiac lesions, whether functional or organic, affect a man's chances of getting work in the labour market and to what extent they are actually prejudicial to him when he does get it.

The progress of modern medicine has stripped heart disease of many of its terrors simply by demonstrating that there are numerous organic affections of the heart which are compatible with a life of reasonable length and of a not too restricted activity. Still, for all that, one must realize that an organic disease of the heart is a handicap in the labour market, more especially as heart disease still connotes to the employer the idea of early and sudden death, as doubtless it did to the profession in the dim and distant past, for the ideas held by doctors in one generation become the idées fixes of the laity in the next. 